\title{
Report of the CCFA Pediatric Bone, Growth and Muscle Health Workshop, New York City, November 11-12, 2011, With Updates
}

\author{
Francisco A. Sylvester, MD, ${ }^{*}$ Catherine M. Gordon, MD, MSC, ${ }^{+}$Meena Thayu, $\mathrm{MD}^{\neq}{ }^{\neq}$ \\ Jon M. Burnham, MD, MSCE, Lee A. Denson, MD, Jonah Essers, MD, MPH, Serge Ferrari, MD," \\ Neera Gupta, MD, MAS, ${ }^{\pi}$ Martin Hewison, PhD, "Sibylle Koletzko, MD, PhD, ${ }^{* *}$ Laura McCabe, PhD, ${ }^{++}$ \\ Helen Pappa, MD, MPH, ${ }^{+}$Ian Sanderson, $M D_{,}^{\neq \neq}$Leanne Ward, MD, PhD, ${ }^{\S \S}$ and Stefano Zanotti, PhD ${ }^{\|\|}$
}

\begin{abstract}
Growth retardation, delayed puberty, decreased bone mass, altered bone architecture, hypovitaminosis D and skeletal muscle mass deficits are common in children with inflammatory bowel diseases. The Crohn's and Colitis Foundation of America sponsored a multidisciplinary workshop on the subject of Bone and Skeletal Growth in Pediatric IBD, held in New York City in November 2011. The topic of the workshop was a key recommendation of the Foundation's Pediatric Challenges meeting in 2005. The Litwin Foundation provided a generous grant to support this crucial research and workshop through the CCFA. The workshop featured 15 presentations by researchers from the United States, Canada, Switzerland, Germany, and the United Kingdom and a number of posters elucidating diverse aspects of the problem of growth retardation and compromised bone health in pediatric Crohn's disease and ulcerative colitis. The workshop comprised original, basic, and clinical research and relevant reviews of underlying genetics, molecular biology, endocrinology, immunology, and bone physiology research. Investigators funded by CCFA and the Litwin Family Foundation are marked by an asterisk after their name in the text. Workshop presentations fell under 3 broad categories: "Mechanisms of Suppression and Growth of Bone Cell Function by Inflammation," "Impact of IBD on Growth and Bone Health," and "Approaches to Address Growth Failure and Low Bone Mass in Children with IBD," summarized herein. We have cited the publications that resulted from this granting mechanism in the appropriate section and references for pertinent updates on each topic.
\end{abstract}

(Inflamm Bowel Dis 2013;19:2919-2926)

Key Words: inflammatory bowel disease, children, bone, osteoporosis, sarcopenia, delayed puberty, osteoblasts, osteoclasts

\section{MECHANISMS OF SUPPRESSION AND GROWTH OF BONE CELL FUNCTION BY INFLAMMATION}

Inflammation, most notably in children with Crohn's disease (CD), can result in growth failure (defined as a growth velocity less than $-2 \mathrm{SD}$ compared with unaffected children) and altered bone remodeling and modeling. In children with new onset

Received for publication April 15, 2013; Accepted July 17, 2013.

From the *Connecticut Children's Medical Center, Hartford, CT and University of Connecticut Health Center, Farmington, CT; †Children's Hospital Boston, Harvard University, Boston, MA; ${ }^{\ddagger}$ Children’s Hospital of Philadelphia, Perelman School of Medicine at the University of Pennsylvania, Philadelphia, PA; ${ }^{\S}$ Cincinnati Children's Hospital Medical Center, Cincinnati, OH; "University Hospital of Geneva, Geneva, Switzerland; “University of California San Francisco Children's Hospital, San Francisco, CA and UCLA/Orthopaedic Hospital, Los Angeles, CA; **Dr. von Haunersches Kinderspital, Ludwig Maximilians Universität München, Germany; ${ }^{\dagger}$ Michigan State University, East Lansing, MI; ${ }^{\text {th }}$ Royal London Hospital, Bart's \& London School of Medicine, London, United Kingdom; ${ }^{\S \S}$ Children's Hospital of Eastern Ontario, Ontario, Canada; and ${ }^{\|\|}$Saint Francis Hospital and Medical Center, Hartford CT and University of Connecticut Health Center, Farmington, CT.

The authors have no conflicts of interest to disclose.

Reprints: Francisco A. Sylvester, MD, Connecticut Children's Medical Center, Division of Digestive Diseases, Hepatology and Nutrition, University of Connecticut Health Center, 282 Washington Street, Hartford, CT 06106 (e-mail: fsylves@ccmckids.org).

Copyright (C) 2013 Crohn's \& Colitis Foundation of America, Inc.

DOI 10.1097/MIB.0b013e3182a5a004

Published online 22 August 2013.
$\mathrm{CD}$, the activity of bone cells, osteoblasts, and osteoclasts is decreased probably due to disease and treatment factors. ${ }^{1,2}$ This accounts for thinner and potentially weaker bones in pediatric $\mathrm{CD}^{1,3}$ One year after diagnosis, $40 \%$ of children may exhibit decreased linear growth velocity and $33 \%$ may have persistent reduction in linear growth 2 years after diagnosis. ${ }^{4}$ Affected children and adolescents can exhibit a lower final height and suboptimal peak bone mass. ${ }^{5}$ The mechanisms underlying these clinical problems are however not fully understood. Recent progress on the subject is the focus of the following oral presentations.

\section{MECHANISMS OF INFLAMMATORY BOWEL DISEASE SUPPRESSION OF SKELETAL GROWTH AND MINERAL DENSITY}

\section{Laura R. McCabe, PhD*}

Dr. McCabe and colleagues treated mice with $1 \%$ to $5 \%$ dextran sulfate sodium (DSS) in drinking water to induce colitis and described changes in linear growth, bone mass and architecture, and bone cell function. In mice that received 5\% DSS, growth was stunted, serum biomarkers of osteoclast and osteoblast activity were significantly decreased, chondrocyte mass was reduced, and bone mass was lower. All abnormalities corrected after DSS was removed and the colon healed suggesting that treating inflammation 
can restore normal growth and bone formation. Remarkably, a subset of mice treated with $1 \%$ DSS did not lose weight yet had active colitis, a reduction in bone mass, decreased bone formation, and suppressed mineral acquisition rate. Serum osteoclast and osteoblast activity markers were consistently low. This suggests that colitis even without significant weight loss is sufficient to reduce bone mass acquisition. Serum tumor necrosis factor alpha (TNF- $\alpha$ ) was higher during colitis and may be responsible, at least in part, for the suppression of bone growth and modeling. However, cytokines may be also produced in the bone microenvironment in response to colonic inflammation, and activated bone marrow cells may directly suppress bone formation. Interestingly, male mice had more severe colitis than females and also showed bone loss where females did not. ${ }^{6}$

\section{Future Directions}

The gut appears to be an important regulator of bone cell function. Dr. McCabe will continue studying sex differences in the severity of colitis and in the response to treatments that aimed at increasing bone mass, such as selected probiotics. ${ }^{7}$

\section{IMMUNOGENETIC DETERMINANTS OF LINEAR GROWTH IN PEDIATRIC INFLAMMATORY BOWEL DISEASE}

\section{Lee A. Denson, MD*}

Currently, there are no adequate clinical prediction tools for high risk of growth failure in children with Inflammatory bowel disease (IBD). Dr. Denson's research seeks to fill this gap and identify the risk factors for growth faltering in 2 cohorts of children with IBD. He is testing 3 hypotheses: (1) linear growth is affected by disease activity and anti-TNF- $\alpha$ therapy; (2) a subset of patients will have growth retardation even in the presence of mild or inactive disease; (3) genetic mutations of nucleotide-binding oligomerization domain 2 and autoantibodies to granulocyte macrophage colony-stimulating factor (GM-CSF) cause intestinal barrier dysfunction and suppression of tissue growth hormone receptors. Dr. Denson has observed high rates of growth failure at IBD diagnosis that persisted 2 years after diagnosis. A modest increase in growth accompanied anti-TNF- $\alpha$ therapy, however below what researchers anticipated. This subset of patients did not achieve their predicted height 2 years after diagnosis. Significantly, the highest degree of growth failure was associated with the presence of GM-CSF autoantibodies and nucleotide-binding oligomerization domain 2 mutations and altered intestinal barrier function ${ }^{8}$ and resistance to growth hormone. ${ }^{9}$ Therefore, therapies directed at improving barrier function may improve the potential for catch up growth in children with IBD. A subset of children with CD and GM-CSF autoantibodies may benefit from GM-CSF therapy. ${ }^{10}$

\section{Future Directions}

To build a model to predict the risk of significant growth failure and validate findings in a replication cohort of three hundred patients from the CCFA RISK Study.

\section{GENETICS OF GROWTH FAILURE IN CD}

\section{Jonah Essers, MD, MPH}

Dr. Jonah Essers submitted that final height of children with IBD is the result of a combination of genetic, disease, and treatment factors. As an example, he discussed Dr. Jessica Lee's study that examined height $z$ scores of 210 children with IBD and their parents. The data showed that parents of non-growthimpaired children with IBD fit the mean $z$ score for adult height, whereas parents of children with growth impairment are also shorter. The height $z$ score of unaffected siblings of children with growth deficits is also shorter than normal. Of the 108 patients with IBD followed into adulthood, mean height $z$ scores are lower than predicted. Taking into account immunomodulators and biologics, the most important predictors of growth deficits are minimum height $z$ score of the patient and height $z$ score of the parents. ${ }^{11}$

\section{Future Directions}

Future directions will address whether genetic factors (1) directly influence the biology of growth in pediatric IBD or (2) worsen the disease behavior, which as a downstream consequence negatively affects growth. Future efforts will need to refine phenotyping of growth to reflect true growth velocity and will likely involve querying the whole genome rather than candidate genes. However, it is unlikely that genetics alone will be used to predict growth failure except in rare patients with a strong Mendelian hit. The CCFA RISK Project will provide a database for evaluation of genetic influences in groups of clinical IBD phenotypes.

\section{VITAMIN D}

Vitamin D increases intestinal calcium absorption, suppresses parathyroid hormone $(\mathrm{PTH})$, and promotes normal bone mineralization, but vitamin $\mathrm{D}$ may also have functions in immune responses, glucose homeostasis, cardiovascular disease, and cancer prevention. ${ }^{12}$ Vitamin $\mathrm{D}$ seems to be a potent modulator of both innate and adaptive immunity. ${ }^{12}$ The vitamin D receptor is present in a wide variety of immune cells, ${ }^{13-18}$ and vitamin $\mathrm{D}$ receptor polymorphisms confer increased risk for CD. ${ }^{19}$

Vitamin D deficiency can stem from low intake, decreased skin production, impaired absorption, obesity, renal, and hepatic failure or loss of 25-hydroxy-vitamin D (25OHD). Winter, aging, skin pigmentation, and use of sun block affect cutaneous synthesis. Certain medications like anticonvulsants, antiretrovirals, corticosteroids, and cyclosporine are associated with vitamin D deficiency. The Institute of Medicine and the Endocrine Society recommend 25OHD testing for those at risk for vitamin D deficiency. ${ }^{20,21}$ Because vitamin $\mathrm{D}$ deficiency is common in children and adolescents with IBD, a serum 25OHD level should be obtained at least yearly. ${ }^{22}$ 


\section{RELEVANCE OF VITAMIN D TO THE INTESTINAL MICROBIOME}

\section{Martin Hewison, PhD}

There are numerous lines of evidence that implicate a potential role for vitamin D deficiency in IBD onset and disease severity. Vitamin D may enhance intestinal innate immunity, thus helping to control the density and diversity of the gut flora. Vitamin D may also prevent chronic intestinal inflammation from taking place by suppressing the activation of the adaptive immune system in the gut. $^{23}$ Unlike the renal $1-\alpha$ hydroxylation of vitamin D by CYP27B1, which is regulated primarily by $\mathrm{PTH}$, the tissue concentration of 1,25-dihydroxy vitamin $\mathrm{D}(1,25$ $\left.(\mathrm{OH})_{2} \mathrm{D}\right)$ is mainly regulated by the availability of $25 \mathrm{OHD}$. This is relevant to IBD because $1,25(\mathrm{OH})_{2} \mathrm{D}$, is a potent stimulator of nucleotide-binding oligomerization domain 2 , an important intracellular microbial recognition receptor ${ }^{24}$ and the production of cathelicidin in macrophages. ${ }^{15}$ Therefore, vitamin D deficiency may result in dysbiosis and contribute to the inflammatory response in IBD. In addition, experimental manipulations that reduce the intestinal concentration of $1,25(\mathrm{OH})_{2} \mathrm{D}$ increase the severity of DSS-induced colitis in mice and produce aberrant innate immune responses (e.g., reduced antimicrobial peptides) to enteric bacteria. ${ }^{25,26}$ These observations show that vitamin D can have an impact on the gut microbiota in several ways: enhanced bacterial killing, improved surveillance of enteric bacteria, altered composition of microbiota, and modulation of adaptive immune components. Dr. Hewison's observations suggest that vitamin D deficiency may be an environmental factor that plays a role in IBD pathogenesis.

In a subset of patients with $\mathrm{CD}$, there may be an over production of $1,25(\mathrm{OH})_{2} \mathrm{D}$, similar to what happens in other granulomatous diseases. ${ }^{27}$ The precise mechanism causing overactivation of vitamin $\mathrm{D}$ and hypercalcemia in $\mathrm{CD}$ is unclear but may involve effects of enteric bacteria acting to enhance the vitamin D system within patient gastrointestinal tissues.

\section{Future Directions}

To determine if vitamin $\mathrm{D}$ supplementation in patients with IBD affects gut immune responses and disease severity.

\section{VITAMIN D IN PEDIATRIC AUTOIMMUNE DISEASES}

\section{Jon M. Burnham, MD, MSCE}

Systemic lupus erythematosus (SLE), like IBD, is a chronic inflammatory disease that can affect children and adults. Like in IBD, there is evidence that vitamin D may help patients with SLE. Low serum vitamin $\mathrm{D}$ is associated with increased disease activity is pediatric ${ }^{28}$ and adult $\mathrm{SLE}^{29}$ suggesting that supplementation of vitamin D may help reduce SLE disease activity. For example, a pilot study in pediatric SLE showed that 15,000 to $30,000 \mathrm{IU}$ vitamin D per week improved 25OHD serum levels without a change evident in serum calcium or urine calcium/creatinine ratio. ${ }^{30}$ However, it has not yet been tested if correcting vitamin $\mathrm{D}$ deficiency in SLE improves disease outcomes.

Interferon alpha (IFN- $\alpha$ )-induced gene expression is a biomarker for measuring disease activity in SLE. ${ }^{31}$ Dr. Burnham chairs a clinical trial funded by the Autoimmunity Centers of Excellence and sponsored by the National Institute of Allergy and Infectious Diseases to determine whether high-dose vitamin D3, compared with standard dose, improves the IFN- $\alpha$ signature in children with SLE and vitamin D deficiency over a 3-month period (NCT01709474). In addition, changes in glucose homeostasis and biomarkers of bone metabolism will be measured.

\section{Future Directions}

In pediatric autoimmune/inflammatory conditions, studies are needed to determine whether vitamin D influences susceptibility, severity, persistence, and tissue damage and whether vitamin D supplementation can optimize clinical outcomes.

\section{BONE CELL METABOLISM AND INFLAMMATION}

Bone tissue is remodeled continuously throughout life by discrete multicellular units, where bone matrix is produced by osteoblasts and resorbed by osteoclasts in a coordinated effort to maintain bone mass, bone architecture, and bone strength. In addition, during growth and development, both osteoblasts and osteoclasts are active in a process called bone modeling. Both bone remodeling and modeling are susceptible to chronic intestinal inflammation. The following section discusses research opportunities in osteoimmunology. ${ }^{32}$

\section{INFLAMMATORY TARGETS IN KEY DEVELOPMENTAL SIGNALS OF OSTEOBLASTS}

\section{Stefano Zanotti, PhD}

Pluripotent mesenchymal cells in the bone marrow can differentiate into osteoblasts, adipocytes, and chondrocytes. Run $\times 2^{33}$ and Osterix ${ }^{34}$ are transcription factors required for osteoblast differentiation, and their expression characterizes cells committed to the osteoblastic lineage. ${ }^{35}$ Differentiation of mesenchymal cells to the osteoblastic lineage is regulated by a network of signaling pathways that includes bone morphogenetic protein/signaling mothers against decapentaplegic (BMP/Smad), and Wnt. ${ }^{36}$ There is growing evidence that inflammation suppresses osteoblast activity and bone formation. However, the mechanisms for this effect are less clear. TNF- $\alpha$ suppresses osteoblastogenesis, possibly by inhibiting the activity of BMP/ Smad $^{37}$ and Wnt. ${ }^{38}$ The Notch pathway may also be affected by inflammatory signals emerging from the diseased intestine, ${ }^{39}$ which may offer an additional explanation for the decreased bone mass observed in pathologies with a prominent inflammatory element, such as CD. 


\section{Future Directions}

Future directions are to investigate the effects of inflammation on the signaling pathways that determine osteoblastogenesis to clarify the connection between inflammatory diseases and the lack of bone formation.

\section{OSTEOCLASTS, INFLAMMATION, AND IBD}

\section{Francisco Sylvester, MD}

RANKL produced by osteoblasts and bone marrow stromal cells is an essential cytokine for osteoclast development. RANKL binds to its receptor RANK on osteoclast precursors and induces osteoclast differentiation and activation. ${ }^{40}$ In addition, costimulation through immunoglobulin-like receptors and their ligands is required to form osteoclasts. ${ }^{41}$ Activated T cells, particularly Th17 cells that have an important role in the pathogenesis of IBD, can produce RANKL. ${ }^{42}$ Cytokines such as TNF- $\alpha$, IL-6, IL-17, and IFN- $\gamma$ that are found in the inflamed intestinal mucosa in IBD may regulate osteoclast development by regulating the RANKL signaling pathway. ${ }^{43,44}$ These cytokines may act on osteocytes that are an important source of RANKL in bone. ${ }^{45,46}$ Moreover, in IBD, osteoclasts may directly respond to environmental signals in the bone microenvironment, including cytokines, chemokines, prostaglandins, and pathogen-associated molecular patterns and become more or less developed and activated in different regions of the bone.

In children with IBD, osteoclast activity seems to be suppressed, at least at diagnosis. However, regional differences in osteoclast function responsible for expanding the bone marrow cavity and lengthening the bones may make long bones thinner and weaker in children with IBD. ${ }^{3}$ The behavior of osteoclasts may also change with therapies such as corticosteroids and biologics and with disease progression.

\section{Future Directions}

Future directions are to better understand the systemic and local signals that control osteoclast differentiation and function in pediatric IBD.

\section{IMPACT OF IBD ON GROWTH AND BONE HEALTH}

The previous discussion underscored several mechanisms by which intestinal inflammation may reduce bone formation and linear growth and augment bone resorption. The purpose of the following section is to understand how IBD impacts linear growth and the skeleton in the clinic and to identify opportunities to improve final adult height and peak bone mass in young patients with IBD.

To understand the effects of IBD on bone, one should consider that bone and skeletal muscle form a functional unit. ${ }^{47}$ Physical activity influences bone metabolism both directly and indirectly. If dynamic strain during exercise exceeds an upper threshold, the bone-forming cells, osteoblasts, are stimulated and form the bone matrix. Weight-bearing activities like gymnastics, running, or jumping activities induce high dynamic loads and therefore enhance bone growth and formation and may be a therapeutic option to enhance bone mass in children with IBD.

\section{THE ROLE OF BONE HISTOMORPHOMETRY IN PEDIATRIC IBD BONE AND GROWTH RESEARCH}

\section{Frank Rauch, MD}

Dr. Rauch and colleagues evaluated static histomorphometry on transiliac bone samples in 20 patients (age range 8.4-17.7 yr, 12 males $/ 8$ females) with newly diagnosed CD and compared the results with reference data from their laboratory. ${ }^{2,48}$ This study revealed a slightly low cortical width but a normal amount of trabecular bone. ${ }^{2}$ However, the percentage of trabecular bone surface covered by osteoid or osteoclasts was low, suggesting that both bone formation and bone resorption were suppressed. Therefore, histomorphometry of transiliac bone biopsies suggests that young patients with $\mathrm{CD}$ have slow trabecular bone turnover at diagnosis.

\section{Future Directions}

Transiliac bone biopsy can be easily performed by trained physicians. Bone histomorphometry should be part of the evaluation of children with low bone mass to better understand how IBD affects bone architecture and bone cell function and direct treatment for low bone mass appropriately.

\section{STRUCTURAL BASES OF BONE FRAGILITY IN YOUNG PATIENTS WITH IBDS}

\section{Serge Ferrari, MD*}

Dr. Ferrari and colleagues recruited patients with $\mathrm{CD}(\mathrm{n}=76)$, ulcerative colitis $(n=29)$, and IBD undefined $(n=3)$ from the Swiss IBD cohort. Dual x-ray absorptiometry (DXA) (Discovery A; Hologic, Bedford, MA) measured areal bone mineral density (BMD) at the distal radius, hip, and spine. The DXA scanner also performed vertebral fracture analysis. High-resolution peripheral quantitated computed tomography (pQCT) (XtremeCT; Scanco, Brüttisellen, Switzerland) determined volumetric bone mineral density and trabecular and cortical bone microstructure at distal radius and tibia. Questionnaires recorded clinical fractures, disease characteristics, and environmental variables. BMD and microstructure were compared between subjects with IBD and 345 age, sex, height, and fracture history matched healthy controls. Dr. Ferrari compared patients who did and did not sustain fractures and adjusted for multiple confounders.

The mean age of IBD subjects was 22.8 years and mean duration of IBD was 6.1 years (range 1.0-22.3 years). Clinical fractures were reported by 39 patients with IBD (36\%; mean age of $12.6 \mathrm{yr}$ at first fracture). The majority of fractures were at the forearm, arm, or hand. Five subjects had morphometric vertebral fractures (grade 1 or 2) and 11 had multiple fractures. Dr. Ferrari 
observed that young subjects with IBD have low bone mineral density and poor bone microarchitecture compared with healthy controls.

\section{Future Directions}

Future studies should examine what therapies for IBD are associated with the highest risk of alterations in bone microarchitecture, particularly in the trabecular bone compartment, which are specifically associated with fractures in patients with IBD.

\section{THE FUNCTIONAL MUSCLE-BONE UNIT IN PEDIATRIC IBD}

\section{Leanne Ward, MD*}

Children with $\mathrm{CD}$ often have a reduction in lean mass (sarcopenia), potentially owing to nutritional compromise and inflammatory mediation by cytokines. Previous studies have suggested that sarcopenia may be responsible for the low bone mass in children with CD. Therefore, Dr. Ward and colleagues examined the "muscle-bone phenotype" in pediatric CD and determined whether sarcopenia of leg muscles is associated with a reduction in leg muscle function and bone deficits. Between 2008 and 2011, she enrolled 67 children (mean age of $13.5 \mathrm{yr}$, mean bone age $13.8 \mathrm{yr}$; $62 \%$ males) with confirmed diagnosis of CD. Over $90 \%$ of the subjects had moderate/severe CD. Dr. Ward used DXA of the spine, metacarpal morphometry for cortical and marrow area evaluation, and pQCT of the tibia to examine trabecular and cortical density, bone mineral content, and tibia muscle cross-sectional area. A dynamic measure of strength, "jumping mechanography" used the Leonardo Force Plate (Novotec Medical GmbH, Pforzheim, Germany), to measure peak jump power per body weight on a single 2-legged jump.

Dr. Ward found that leg muscle mass at the tibia was significantly reduced in children with newly diagnosed CD compared with controls, along with a significant reduction in peak jump power. Although both muscle mass and bone mass were reduced, the amount of bone was appropriate for the amount of muscle mass. Dr. Ward also observed a high cortical density of long bones, probably secondary to low intracortical remodeling activity.

\section{Future Directions}

Sarcopenia in children with $\mathrm{CD}$ is associated with decreased muscle power and strength and reduced bone mass. Future studies should assess the impact of exercise programs on muscle mass, strength, and power in children with IBD.

\section{Sibylle Koletzko, MD}

Dr. Koletzko and colleagues performed a longitudinal study in a large cohort of children with IBD where she measured BMD and muscle mass with pQCT of the lower arm on a yearly basis, some starting at the time of diagnosis and others later in the disease course. Newly diagnosed patients had the lowest $z$ scores for muscle mass. Muscle mass $z$ score improved over time, but it did not normalize over the duration of the study in children with IBD. ${ }^{4-51}$ Dr. Koletzko hypothesized that bone adapts to the smaller muscle mass in pediatric IBD, resulting in a low bone mass, bone strength, and different bone geometry as compared with healthy children.

Dr. Koletzko also examined the grip force, number of daily steps, and energy expenditure over 3 days in 39 children with IBD compared with age-based and sex-based controls. Grip force and number of steps were lower in the patient group. Patients with IBD tended to have more sedated daily activity and more sleep time. ${ }^{51}$

Dr. Koletzko presented another study of trabecular and cortical BMD in children with CD treated with exclusive enteral nutrition over 8 weeks followed by stepwise introduction of a regular diet. The muscle mass of the patients with CD improved dramatically with exclusive enteral nutrition. Cortical BMD decreased and trabecular BMD increased, suggesting activation of bone turnover with enteral nutrition. However, the gains incurred during exclusive enteral feeding therapy were not sustained after a normal diet was resumed. ${ }^{50,51}$

\section{Future Directions}

Future studies should evaluate whether bone strength in children with IBD can be improved by physical activity programs that increase muscle mass, especially during puberty when most bone mass normally accumulates.

\section{SEX DIFFERENCES IN STATURAL GROWTH IMPAIRMENT IN PEDIATRIC PATIENTS WITH CD}

\section{Neera Gupta, MD, MAS}

Dr. Gupta and colleagues studied 989 pediatric patients with CD enrolled in the Pediatric IBD Consortium Registry. She found that although the overall course of disease was more severe in females, ${ }^{52,53}$ the cumulative incidence of growth failure was greater in males. ${ }^{53}$ These data suggest that the negative impact of disease severity on linear growth differs by sex.

Dr. Gupta also reported on a cross-sectional study of linear growth in 82 patients with CD (43\% female) younger than 21 years of age followed between 2007 and $2009 .{ }^{54}$ She observed that serum insulin-like growth factor 1 (IGF-1) adjusted to chronological age and bone age $z$ scores were lower in males than females. Lower IGF-1 in males may explain sex differences in growth impairment in CD. Inflammation appears to more adversely affect hormone levels and linear growth in males.

\section{Future Directions}

Prospective longitudinal studies are needed to clarify the etiology of sex differences in growth impairment in pediatric CD.

\section{APPROACHES TO ADDRESS GROWTH FAILURE AND LOW BONE MASS IN CHILDREN WITH IBD}

Pediatric IBD is associated with low bone remodeling and modeling and sarcopenia due to disease and treatment factors. The 
inflammation caused by IBD in children may have long-term effects on bone health, adult height, muscle strength, and maturation if not recognized and treated. In this session, inflammation, malnutrition (with associated reductions in IGF-1), and vitamin D deficiency were explored as therapeutic targets for intervention for children with $\mathrm{CD}$ and ulcerative colitis.

\section{CHANGES IN BONE AND MUSCLE AND MUSCLE MASS AFTER TNF-ALPHA INHIBITION IN PEDIATRIC CD}

\section{Meena Thayu, MD, MSCE}

Dr. Thayu and colleagues described changes in bone turnover markers during infliximab induction therapy in pediatric $\mathrm{CD}$ and identified determinants of 12-month improvements in bone and muscle mass after initiation of this therapy. The study prospectively enrolled 74 subjects (63\% male, 5-21 yr of age) initiating infliximab for the first time. The pQCT measurements were converted to race-specific $z$ scores. Trabecular and cortical BMD were determined relative to age. Cortical dimensions and muscle area relative to tibia length were adjusted for age. Bone biomarkers were converted to Tanner stage-specific $z$ scores.

Infliximab therapy was associated with marked increase in the bone formation marker bone-specific alkaline phosphatase at 10 weeks and with improvements in height $z$ score at 54 weeks. ${ }^{55}$ Trabecular BMD increased, cortical BMD decreased, and endocortical bone recovered, changes consistent with new bone formation. Greater height velocity was associated with greater changes in volumetric BMD, endosteal, and muscle cross-sectional area $z$ scores. Deficits in periosteal and section modulus persisted, despite other improvements. ${ }^{56}$

The results indicate that recovery of linear growth, bone density, bone dimensions and strength, and muscle mass in patients with $\mathrm{CD}$ treated with infliximab is most likely to occur in younger that have growth potential.

\section{Future Directions}

The next set of analyses will incorporate measures of physical activity, dietary intake, osteoprotegerin/receptor activator of nuclear factor $\kappa \mathrm{B}$, cytokine and growth factor levels, vitamin $\mathrm{D}$, and PTH levels.

\section{PHARMACOKINETIC STUDIES OF RECOMBINANT HUMAN IGF-1 IN CHILDREN WITH CD-INDUCED GROWTH RETARDATION}

\section{lan Sanderson, MD*}

The best treatment for growth failure in CD is treatment of inflammation; however, there are cases where the use of recombinant human IGF-1 may be useful to treat poor growth. This study is aimed at developing a pharmacokinetic model for safe and effective dosing of recombinant IGF-1 in children with CD.
In a rat model of colitis, Dr. Ian Sanderson and colleagues established that inflammation by itself can suppress linear growth, independent of malnutrition. ${ }^{57}$ IGF-1 is depressed in rats with colitis, and injections of IGF-1 help to restore linear growth. This finding stimulated Dr. Sanderson to consider the use of IGF-1 in children with $\mathrm{CD}$ with active inflammation, despite therapy and poor growth. A first step was to study the pharmacokinetics of IGF-1 in children with CD. Dr. Sanderson and colleagues designed a study of 8 children with $\mathrm{CD}$ to determine the optimal dosing model for IGF-1. Each child was hospitalized twice with informed consent. Each child had a first dose of IGF-1, and levels were followed over 24 hours. Three months later, each child was admitted for 5 days, and 12-hour dosing took place on alternate days. Although some children with active $\mathrm{CD}$ had protein-losing enteropathy, intestinal losses did not affect the rate of IGF-1 decline after dosing. This dosing model takes into account patient weight and disease activity and is able to restore normal circulating levels of IGF-1 in children with CD receiving recombinant IGF-1 therapeutically.

\section{Future directions}

A long-term study to evaluate the safety and efficacy of IGF-1 for growth enhancement in children with growth delay who are resistant to anti-inflammatory medications.

\section{OPTIMIZATION OF VITAMIN D STATUS AND ITS IMPACT ON BONE AND HEALTH AND DISEASE OUTCOMES IN YOUNG PATIENTS WITH IBD}

\section{Helen Pappa, MD, MPH}

Dr. Pappa reported on a study comparing patients with IBD to healthy adolescents, where $34.6 \%$ of patients with IBD had $25 \mathrm{OHD}<15 \mathrm{ng} / \mathrm{mL}$ versus $24 \%$ in healthy adolescents, at the same center. ${ }^{58}$ Risk factors for low 25OHD in children with IBD included winter season, dark skin, low BMI $z$ score, low serum albumin, high ESR, corticosteroid exposure, and upper gastrointestinal disease.

Dr. Pappa and colleagues went on to examine the efficacy and safety of 3 regimens to treat hypovitaminosis D (25OHD level $\leq 20 \mathrm{ng} / \mathrm{mL}$ ) in patients 5 to 21 years with IBD: 2000 IU D2 per day; 2000 IU D3 per day, and 50,000 IU D2 per week all administered orally for 6 weeks, each in 20 subjects allocated randomly. All patients also received 800 or $1200 \mathrm{mg}$ of calcium per day. There were no serious adverse events. The highest dose of vitamin D (50,000 IU/wk) was the most effective to raise serum $25 \mathrm{OHD}$ and should therefore be the regimen of choice to treat hypovitaminosis D in children with IBD. ${ }^{58}$ Interestingly, serum PTH response was blunted in children with IBD for unknown mechanisms that may have to do with relative insensitivity of the calcium sensing receptor in the parathyroid gland in IBD.

Dr. Pappa and colleagues also evaluated 2 maintenance regimens, each including 30 patients assigned randomly: 400 IU D2 per day for 1 year or 2000 IU D2 per day from November to 
April and 1000 IU per day from May to December. Neither regimen maintained the serum $25 \mathrm{OHD}>32 \mathrm{ng} / \mathrm{mL}$ throughout the year and were only $65 \%$ to $68 \%$ effective to keep $25 \mathrm{OHD}$ $>20 \mathrm{ng} / \mathrm{mL}$ throughout the year. The higher dose regimen was associated with a slightly higher mean $25 \mathrm{OHD}$ level. Serum IL-6 was lower in subjects receiving the higher dose of vitamin $\mathrm{D}{ }^{58}$ None of the regimens was associated with improved BMD scores.

\section{Future Directions}

To determine a target serum 250HD level that results in optimal bone health and improved disease outcomes and vitamin D supplementation regimens that enable the target level. The mechanisms behind the blunted PTH response to low vitamin D need to be examined. The role of vitamin $\mathrm{D}$ analog or other vitamin $\mathrm{D}$ receptor agonists in management of inflammation could also be an opportunity for investigation.

\section{SUMMARY AND CONCLUSIONS}

Pediatric IBD, especially CD, is associated with significant and persistent alterations in bone mass and architecture and in skeletal muscle mass and strength. Suppression of osteoblast (and perhaps osteocyte) function and regional upregulation of osteoclast function by inflammatory factors play significant roles. It is possible that intestinal inflammation affects the regenerative capacity of muscle stem cells. Therefore, the functional bonemuscle unit is impaired in pediatric IBD. Routinely obtaining accurate height and weight, Tanner stage, and menstrual history is essential to identify children whose growth and pubertal progression are delayed. Current technologies including DXA, pQCT, and bone histomorphometry of transiliac bone biopsy can accurately characterize the impact of IBD on bone and muscle in individual patients. Certain treatments such as TNF- $\alpha$ blockers and enteral nutritional therapy offer promise to increase bone formation and linear growth. These observations need to be confirmed, and additional therapies need to be identified that achieve the same anabolic effects on bone, muscle, and growth in pediatric IBD. Adequate dietary intake of calcium and maintenance of serum $25 \mathrm{OHD}>20 \mathrm{ng} / \mathrm{mL}$ should ensure sufficient substrate to mineralize bone adequately. This goal may be difficult to achieve in some children with IBD. Patients whose growth failure persists despite adequate disease control or in whom disease control is difficult to achieve with current therapies may be candidates for treatment with growth hormone or IGF-1 if there is growth hormone resistance. When designing therapies to improve bone mass and linear growth, important differences between males and females should be taken into account. Despite excellent progress obtained as a consequence of this CCFA funding mechanism, many key questions remain. Children afflicted by IBD and their families eagerly await the results of new research.

\section{REFERENCES}

1. Sylvester FA, Wyzga N, Hyams JS, et al. Natural history of bone metabolism and bone mineral density in children with inflammatory bowel disease. Inflamm Bowel Dis. 2007;13:42-50.
2. Ward LM, Rauch F, Matzinger MA, et al. Iliac bone histomorphometry in children with newly diagnosed inflammatory bowel disease. Osteoporos Int. 2010;21:331-337.

3. Dubner SE, Shults J, Baldassano RN, et al. Longitudinal assessment of bone density and structure in an incident cohort of children with Crohn's disease. Gastroenterology. 2009;136:123-130.

4. Griffiths AM, Nguyen P, Smith C, et al. Growth and clinical course of children with Crohn's disease. Gut. 1993;34:939-943.

5. Markowitz J, Grancher K, Rosa J, et al. Growth failure in pediatric inflammatory bowel disease. J Pediatr Gastroenterol Nutr. 1993;16:373-380.

6. Harris L, Senagore P, Young VB, et al. Inflammatory bowel disease causes reversible suppression of osteoblast and chondrocyte function in mice. Am J Physiol Gastrointest Liver Physiol. 2009;296:G1020-G1029.

7. McCabe LR, Irwin R, Schaefer L, et al. Probiotic use decreases intestinal inflammation and increases bone density in healthy male but not female mice. J Cell Physiol. 2013;228:1793-1798.

8. Nylund CM, D'mello S, Kim MO, et al. Granulocyte macrophage-colonystimulating factor autoantibodies and increased intestinal permeability in Crohn disease. J Pediatr Gastroenterol Nutr. 2011;52:542-548.

9. D'Mello S, Trauernicht A, Ryan A, et al. Innate dysfunction promotes linear growth failure in pediatric Crohn's disease and growth hormone resistance in murine ileitis. Inflamm Bowel Dis. 2012;18:236-245.

10. Roth L, MacDonald JK, McDonald JW, et al. Sargramostim (GM-CSF) for induction of remission in Crohn's disease: a cochrane inflammatory bowel disease and functional bowel disorders systematic review of randomized trials. Inflamm Bowel Dis. 2012;18:1333-1339.

11. Lee JJ, Escher JC, Shuman MJ, et al. Final adult height of children with inflammatory bowel disease is predicted by parental height and patient minimum height Z-score. Inflamm Bowel Dis. 2010;16:1669-1677.

12. Hewison M. Vitamin D and the immune system: new perspectives on an old theme. Rheum Dis Clin North Am. 2012;38:125-139.

13. Griffin MD, Lutz W, Phan VA, et al. Dendritic cell modulation by 1alpha,25 dihydroxyvitamin D3 and its analogs: a vitamin D receptordependent pathway that promotes a persistent state of immaturity in vitro and in vivo. Proc Natl Acad Sci U S A. 2001;98:6800-6805.

14. Hewison M, Freeman L, Hughes SV, et al. Differential regulation of vitamin $\mathrm{D}$ receptor and its ligand in human monocyte-derived dendritic cells. J Immunol. 2003;170:5382-5390.

15. Gombart AF, Borregaard N, Koeffler HP. Human cathelicidin antimicrobial peptide (CAMP) gene is a direct target of the vitamin D receptor and is strongly up-regulated in myeloid cells by 1,25-dihydroxyvitamin D3. FASEB J. 2005;19:1067-1077.

16. Yu S, Cantorna MT. The vitamin D receptor is required for iNKT cell development. Proc Natl Acad Sci U S A. 2008;105:5207-5212.

17. Wu S, Liao AP, Xia $\mathrm{Y}$, et al. Vitamin $\mathrm{D}$ receptor negatively regulates bacterial-stimulated NF-\{kappa\}B activity in intestine. Am J Pathol. 2010;177:686-697.

18. von Essen MR, Kongsbak M, Schjerling P, et al. Vitamin D controls T cell antigen receptor signaling and activation of human $\mathrm{T}$ cells. Nat Immunol. 2010;11:344-349.

19. Simmons JD, Mullighan C, Welsh KI, et al. Vitamin D receptor gene polymorphism: association with Crohn's disease susceptibility. Gut 2000;47:211-214.

20. IOM (Institute of Medicine). Dietary reference intakes for calcium and vitamin D. Washington, DC: The National Academies Press; 2011.

21. Holick MF, Binkley NC, Bischoff-Ferrari HA, et al. Evaluation, treatment, and prevention of vitamin D deficiency: an Endocrine Society Clinical Practice Guideline. J Clin Endocrinol Metab. 2011;96:1911-1930.

22. Pappa H, Thayu M, Sylvester F, et al. Skeletal health of children and adolescents with inflammatory bowel disease. J Pediatr Gastroenterol Nutr. 2011;53:11-25.

23. Ryz NR, Patterson SJ, Zhang Y, et al. Active vitamin D (1,25dihydroxyvitamin D3) increases host susceptibility to Citrobacter rodentium by suppressing mucosal Th17 responses. Am J Physiol Gastrointest Liver Physiol. 2012;303:G1299-G1311.

24. Wang TT, Dabbas B, Laperriere D, et al. Direct and indirect induction by $1,25-$ dihydroxyvitamin $\mathrm{D} 3$ of the NOD2/CARD15-defensin beta2 innate immune pathway defective in Crohn disease. J Biol Chem. 2010;285:2227-2231.

25. Liu N, Nguyen L, Chun RF, et al. Altered endocrine and autocrine metabolism of vitamin D in a mouse model of gastrointestinal inflammation. Endocrinology. 2008;149:4799-4808. 
26. Lagishetty V, Misharin AV, Liu NQ, et al. Vitamin D deficiency in mice impairs colonic antibacterial activity and predisposes to colitis. Endocrinology. 2010;151:2423-2432.

27. Abreu MT, Kantorovich V, Vasiliauskas EA, et al. Measurement of vitamin D levels in inflammatory bowel disease patients reveals a subset of Crohn's disease patients with elevated 1,25-dihydroxyvitamin D and low bone mineral density. Gut. 2004;53:1129-1136.

28. Wright TB, Shults J, Leonard MB, et al. Hypovitaminosis D is associated with greater body mass index and disease activity in pediatric systemic lupus erythematosus. J Pediatr. 2009;155:260-265.

29. Amital H, Szekanecz Z, Szucs G, et al. Serum concentrations of $25-\mathrm{OH}$ vitamin $\mathrm{D}$ in patients with systemic lupus erythematosus (SLE) are inversely related to disease activity: is it time to routinely supplement patients with SLE with vitamin D? Ann Rheum Dis. 2010;69:1155-1157.

30. von Scheven E, Burnham JM. Vitamin D supplementation in the pediatric rheumatology clinic. Curr Rheumatol Rep. 2011;13:110-116.

31. Kirou KA, Lee C, George S, et al. Activation of the interferon-alpha pathway identifies a subgroup of systemic lupus erythematosus patients with distinct serologic features and active disease. Arthritis Rheum. 2005;52:1491-1503.

32. Takayanagi H. New developments in osteoimmunology. Nat Rev Rheumatol. 2012;8:684-689.

33. Takarada T, Hinoi E, Nakazato R, et al. An analysis of skeletal development in osteoblast- and chondrocyte-specific Runx2 knockout mice. J Bone Miner Res. 2013;28:2064-2069.

34. Nakashima K, Zhou X, Kunkel G, et al. The novel zinc finger-containing transcription factor osterix is required for osteoblast differentiation and bone formation. Cell. 2002;108:17-29.

35. Canalis E, Parker K, Feng JQ, et al. Osteoblast lineage-specific effects of notch activation in the skeleton. Endocrinology. 2013;154:623-634.

36. Canalis E. Growth factor control of bone mass. J Cell Biochem. 2009;108: 769-777.

37. Guo R, Yamashita M, Zhang Q, et al. Ubiquitin ligase Smurf1 mediates tumor necrosis factor-induced systemic bone loss by promoting proteasomal degradation of bone morphogenetic signaling proteins. J Biol Chem. 2008;283:23084-23092.

38. Heiland GR, Zwerina K, Baum W, et al. Neutralisation of Dkk-1 protects from systemic bone loss during inflammation and reduces sclerostin expression. Ann Rheum Dis. 2010;69:2152-2159.

39. Zanotti S, Canalis E. Notch regulation of bone development and remodeling and related skeletal disorders. Calcif Tissue Int. 2012;90:69-75.

40. Kong YY, Yoshida H, Sarosi I, et al. OPGL is a key regulator of osteoclastogenesis, lymphocyte development and lymph-node organogenesis. Nature. 1999;397:315-323.

41. Mori Y, Tsuji S, Inui M, et al. Inhibitory immunoglobulin-like receptors LILRB and PIR-B negatively regulate osteoclast development. J Immunol. 2008;181:4742-4751.

42. Kikuta J, Wada Y, Kowada T, et al. Dynamic visualization of RANKL and Th17-mediated osteoclast function. J Clin Invest. 2013;123:866-873.
43. Kong YY, Feige U, Sarosi I, et al. Activated T cells regulate bone loss and joint destruction in adjuvant arthritis through osteoprotegerin ligand. Nature. 1999;402:304-309.

44. Onal M, Xiong J, Chen X, et al. Receptor activator of nuclear factor kappaB ligand (RANKL) protein expression by B lymphocytes contributes to ovariectomy-induced bone loss. J Biol Chem. 2012;287:29851-29860.

45. Nakashima T, Hayashi M, Fukunaga T, et al. Evidence for osteocyte regulation of bone homeostasis through RANKL expression. Nat Med. 2011;17:1231-1234

46. Xiong J, Onal M, Jilka RL, et al. Matrix-embedded cells control osteoclast formation. Nat Med. 2011;17:1235-1241.

47. Schonau E, Werhahn E, Schiedermaier U, et al. Influence of muscle strength on bone strength during childhood and adolescence. Horm Res. 1996;45(suppl 1):63-66.

48. Glorieux FH, Travers R, Taylor A, et al. Normative data for iliac bone histomorphometry in growing children. Bone. 2000;26:103-109.

49. Bechtold S, Alberer M, Arenz T, et al. Reduced muscle mass and bone size in pediatric patients with inflammatory bowel disease. Inflamm Bowel Dis. 2009;16:2016-2227.

50. Werkstetter KJ, Pozza SB, Filipiak-Pittroff B, et al. Long-term development of bone geometry and muscle in pediatric inflammatory bowel disease. Am J Gastroenterol. 2011;106:988-998.

51. Werkstetter KJ, Ullrich J, Schatz SB, et al. Lean body mass, physical activity and quality of life in paediatric patients with inflammatory bowel disease and in healthy controls. J Crohns Colitis. 2012;6:665-673.

52. Gupta N, Cohen SA, Bostrom AG, et al. Risk factors for initial surgery in pediatric patients with Crohn's disease. Gastroenterology. 2006;130: 1069-1077.

53. Gupta N, Bostrom AG, Kirschner BS, et al. Gender differences in presentation and course of disease in pediatric patients with Crohn disease. Pediatrics. 2007;120:e1418-e1425.

54. Gupta N, Lustig RH, Kohn MA, et al. Sex differences in statural growth impairment in Crohn's disease: role of IGF-1. Inflamm Bowel Dis. 2011; $17: 2318-2325$.

55. Thayu M, Leonard MB, Hyams JS, et al. Improvement in biomarkers of bone formation during infliximab therapy in pediatric Crohn's disease: results of the REACH study. Clin Gastroenterol Hepatol. 2008;6:1378-1384.

56. Thayu M, Denson LA, Shults J, et al. Determinants of Changes in linear growth and body composition in incident pediatric Crohn's disease. Gastroenterology. 2010;139:430-438.

57. Sawczenko A, Azooz O, Paraszczuk J, et al. Intestinal inflammationinduced growth retardation acts through IL-6 in rats and depends on the -174 IL-6 G/C polymorphism in children. Proc Natl Acad Sci U S A. 2005;102:13260-13265.

58. Pappa HM, Mitchell PD, Jiang H, et al. Treatment of vitamin D insufficiency in children and adolescents with inflammatory bowel disease: a randomized clinical trial comparing three regimens. J Clin Endocrinol Metab. 2012;97:2134-2142. 